University of San Diego

Digital USD

2010

\title{
On the Structure and Dynamics of Sheared and Rotating Turbulence: Anisotropy Properties and Geometrical Scale- Dependent Statistics
}

\author{
Frank G. Jacobitz \\ University of San Diego, jacobitz@sandiego.edu \\ Kai Schneider \\ Université d'Aix-Marseille \\ Wouter J.T. Bos \\ Université de Lyon \\ Marie Farge \\ Ecole Normale Supérieure
}

Follow this and additional works at: https://digital.sandiego.edu/engineering_facpub

\section{Digital USD Citation}

Jacobitz, Frank G.; Schneider, Kai; Bos, Wouter J.T.; and Farge, Marie, "On the Structure and Dynamics of Sheared and Rotating Turbulence: Anisotropy Properties and Geometrical Scale-Dependent Statistics" (2010). School of Engineering: Faculty Scholarship. 1.

https://digital.sandiego.edu/engineering_facpub/1

This Article is brought to you for free and open access by the School of Engineering at Digital USD. It has been accepted for inclusion in School of Engineering: Faculty Scholarship by an authorized administrator of Digital USD. For more information, please contact digital@sandiego.edu. 


\title{
On the Structure and Dynamics of Sheared and Rotating Turbulence: Anisotropy Properties and Geometrical Scale-Dependent Statistics
}

\author{
Abstract \\ This study is based on a series of nine direct numerical simulations of homogeneous turbulence, in which \\ the rotation ratio $f / S$ of Coriolis parameter to shear rate is varied. The presence of rotation stabilizes the \\ flow, except for a narrow range of rotation ratios $0<<e m>f / S$ \\ Keywords \\ Anisotropy, Turbulent flows, Tensor methods, Vortex dynamics, Reynolds stress modeling
}




\title{
On the structure and dynamics of sheared and rotating turbulence: Anisotropy properties and geometrical scale-dependent statistics
}

\author{
Frank G. Jacobitz, ${ }^{1,2}$ Kai Schneider ${ }^{2,3}$ Wouter J. T. Bos, ${ }^{4}$ and Marie Farge ${ }^{5}$ \\ ${ }^{1}$ Mechanical Engineering Program, University of San Diego, San Diego, California 92110, USA \\ ${ }^{2}$ Laboratoire de Mécanique, Modélisation, et Procédés Propres, Centre National de la Recherche Scientifique, \\ Aix-Marseille Université and Ecole Centrale de Marseille, 38 Rue Joliot-Curie, \\ 13451 Marseille Cedex 20, France \\ ${ }^{3}$ Centre de Mathématiques et d'Informatique, Université de Provence, 39 Rue Joliot-Curie, \\ 13453 Marseille Cedex 13, France \\ ${ }^{4}$ Laboratoire de Mécanique des Fluides et d'Acoustique, Centre National de la Recherche Scientifique, \\ Ecole Centrale de Lyon, Université de Lyon, 69134 Ecully Cedex, France \\ ${ }^{5}$ Laboratoire de Météorologie Dynamique, Centre National de la Recherche Scientifique, \\ Ecole Normale Supérieure, 24 Rue Lhomond, 75231 Paris Cedex 5, France
}

(Received 10 July 2009; accepted 14 May 2010; published online 2 August 2010)

This study is based on a series of nine direct numerical simulations of homogeneous turbulence, in which the rotation ratio $f / S$ of Coriolis parameter to shear rate is varied. The presence of rotation stabilizes the flow, except for a narrow range of rotation ratios $0<f / S<1$. The main mechanism for the flow's destabilization is an increased turbulence production due to increased anisotropy. Reynolds stress and the dissipation rate anisotropy tensors have been evaluated and provide a reference for newly defined anisotropy measures. Wavelet-based directional energies capture the properties of velocity gradients. The intermittency of the flow in different directions is quantified with scale-dependent directional flatness. Scale-dependent helicity probability distribution functions allow one to statistically characterize the geometry of the motion at different scales. Small scales are found locally to be predominantly helical, while large scales are not since they tend to two-dimensionalization for cases with growing turbulent kinetic energy. Joint probability distribution functions show that the signs of velocity helicity and vorticity helicity are strongly correlated. This indicates that vorticity helicity tends to diminish velocity helicity. (C) 2010 American Institute of Physics. [doi:10.1063/1.3457167]

\section{INTRODUCTION}

Rotation and shear are important features of many geophysical and engineering flows (see, for example, Ref. 1). Direct numerical simulations with constant vertical shear $S$ $=\partial U_{1} / \partial x_{2}$ and system rotation with constant Coriolis parameter $f=2 \Omega$ are considered in this study. The rotation axis is perpendicular to the plane of shear and points in the spanwise direction $x_{3}$. It is therefore parallel or antiparallel to the mean flow vorticity. The Cartesian coordinates $\mathbf{x}=(x, y, z)$ $=\left(x_{1}, x_{2}, x_{3}\right)$ refer to the streamwise, vertical, and spanwise directions, respectively. A schematic of the mean flow configuration is shown in Fig. 1.

In the previous studies of Bradshaw ${ }^{2}$ and Tritton, ${ }^{3}$ the effect of rotation was found to be destabilizing in the antiparallel configuration with $0<f / S<1$ and stabilizing otherwise. A detailed discussion of the roles of the rotation ratio $f / S$ and the Bradshaw number $B=f / S(f / S-1$ ) (sometimes called "rotational Richardson number") can be found in Cambon et al. ${ }^{4}$ and Leblanc and Cambon. ${ }^{5}$ It was found that $B$ is not sufficient to characterize the dynamics of the flow. ${ }^{6}$ The neutral cases with $f / S=0$ (pure shear) and $f / S=1$ (zero absolute vorticity) are described by the same Bradshaw number $B=0$, but their dynamics show important differences. Comprehensive investigations of this flow include the works by Salhi and Cambon, ${ }^{6}$ Brethouwer, ${ }^{7}$ and Jacobitz et al. ${ }^{8}$ The studies are complementary as different techniques are employed and a variety of parameter regimes are considered. Rotating sheared turbulence has also been investigated in the context of stratification and magnetohydrodynamics. Linear theory has been used by Salhi ${ }^{9}$ and Kassinos et al. ${ }^{10}$ to compare the effects of rotation and stratification in such flows. Kassinos et al. ${ }^{11}$ investigated passive scalar transport in rotating sheared magnetohydrodynamic turbulence. An overview on homogeneous turbulence dynamics including shear flows can be found in a recent monograph by Sagaut and Cambon. ${ }^{12}$

The aim of this study is an investigation of the anisotropy properties of homogeneous turbulence with shear and rotation. In particular, well-established anisotropy measures, such as the Reynolds stress and dissipation rate anisotropy tensors (see, for example, Ref. 13), are compared to waveletbased measures of anisotropy recently introduced by Bos et al. ${ }^{14}$ (for a recent review of wavelet methods, see Ref. 15). Directional energies and the corresponding spatial fluctuations can be quantified using the orthogonal wavelet decomposition. The conventional anisotropy measures are widely used in the community and provide a reference point for the interpretation of newly defined quantities.

In addition, decompositions of the Reynolds stress anisotropy tensor are considered in this study. Based on the energy, helicity, and polarization decomposition of the three- 


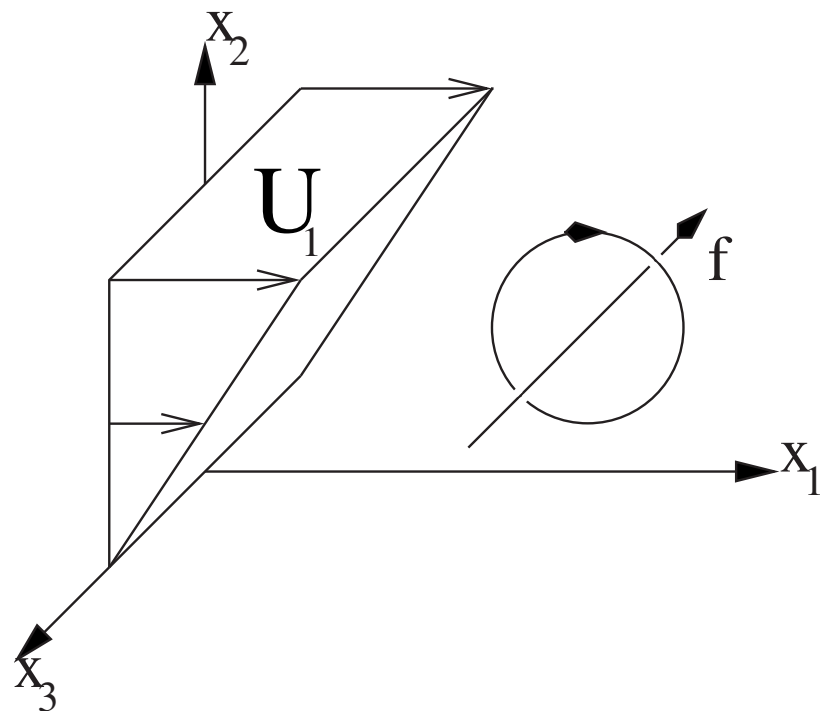

FIG. 1. Schematic of the mean flow configuration with uniform vertical shear $S=\partial U_{1} / \partial x_{2}$ and rotation $f=2 \Omega$. Note that this schematic shows a parallel configuration with negative rotation ratio $f / S$.

dimensional energy spectrum tensor developed in Cambon and Jacquin, ${ }^{16}$ a decomposition of the Reynolds stress anisotropy tensor into directional anisotropy and polarization anisotropy was introduced, first for rotating homogeneous turbulence $^{17}$ and then for general anisotropic turbulence. ${ }^{18}$ In Refs. 19 and 20, Kassinos et al. proposed single-point turbulence structure tensors. The structure dimensionality anisotropy tensor and circulicity anisotropy tensor also decompose the Reynolds stress anisotropy tensor. The measures developed by Kassinos et $a l .{ }^{20}$ and Cambon et al. ${ }^{18}$ can be expressed as linear combinations of each other.

Another goal is the study of scale-dependent statistics, such as flatness, and geometrical quantities, such as helicity probability distribution functions. Directional flatness allows one to quantify the influence of the rotation rate on the flow intermittency. Helicity measures the alignment between the velocity and vorticity vectors and thus characterizes the presence or absence of helical motion. The local scale-dependent helicity has recently been analyzed for forced isotropic turbulence by Yoshimatsu et al. ${ }^{21}$

In the next section, governing equations and numerical approach are introduced. Then results from a series of simulations of rotating and sheared homogeneous turbulence are presented including the turbulence evolution, a comparison of conventional and novel wavelet-based anisotropy measures, turbulence structure tensors, and scale-dependent geo- metrical statistics. In particular, we will assess which characteristics distinguish the flows in which the kinetic energy grows from those in which it decays. Finally, conclusions of the present work are given.

\section{GOVERNING EQUATIONS AND NUMERICAL APPROACH}

The direct numerical simulations performed here are based on the continuity equation for an incompressible fluid and the unsteady three-dimensional Navier-Stokes equation. The following equations are used to determine the fluctuating velocities $\boldsymbol{u}=(u, v, w)=\left(u_{1}, u_{2}, u_{3}\right)$ :

$$
\begin{aligned}
& \nabla \cdot \boldsymbol{u}=0, \\
& \frac{\partial \boldsymbol{u}}{\partial t}+\boldsymbol{u} \cdot \nabla \boldsymbol{u}+S x_{2} \frac{\partial \boldsymbol{u}}{\partial x_{1}}+S u_{2} \boldsymbol{e}_{1}+2 \boldsymbol{\Omega} \times \boldsymbol{u} \\
& \quad=-\frac{1}{\rho_{0}} \nabla p+\nu \nabla^{2} \boldsymbol{u} .
\end{aligned}
$$

Here $p$ contains the pressure and centrifugal force, $\rho_{0}$ is the density, $\nu$ is the kinematic viscosity, and $\boldsymbol{e}_{1}$ is the unit vector in the downstream direction.

In the direct numerical approach, all dynamically active scales of the velocity field are resolved. The above equations are solved in a frame of reference moving with the mean sheared flow (see Ref. 22). This approach allows the application of periodic boundary conditions for the fluctuating components of the velocity field. A spectral collocation method is used for the spatial discretization and the solution is advanced in time with a fourth-order Runge-Kutta scheme. The simulations are performed on a parallel computer using $256 \times 256 \times 256$ grid points. The simulations analyzed in this study are identical to the ones reported in Jacobitz et al. ${ }^{8}$

In the following, results of nine simulations of rotating sheared turbulence are presented and the rotation ratio $f / S$ is varied from -10 to 10 . Negative values of $f / S$ correspond to a parallel configuration and positive values correspond to an antiparallel configuration between the system rotation and the mean flow vorticity. Isotropic turbulence fields are used to initialize all simulations. The values of the initial Taylor microscale Reynolds number $\operatorname{Re}_{\lambda}=45$ and the initial shear number $S K / \epsilon=2$ are identical for all cases. The evolution of the Taylor microscale Reynolds number depends on the fate of the turbulence and reaches values as high as $\operatorname{Re}_{\lambda}=120$. The shear number varies only weakly with $f / S$ and assumes a value of about $S K / \epsilon=6$ in the simulations. This suggests

TABLE I. Properties of the simulations at nondimensional time $\mathrm{St}=5$.

\begin{tabular}{lccccc}
\hline \hline Case & Configuration & $\operatorname{Re}_{\lambda}$ & $S K / \epsilon$ & $\gamma$ & Fate \\
\hline$f / S=-5$ & Parallel & 37.22 & 5.846 & -0.2176 & Decay \\
$f / S=-0.5$ & Parallel & 42.52 & 4.094 & -0.1406 & Decay \\
$f / S=0$ & Shear only & 72.15 & 4.817 & 0.1338 & Growth \\
$f / S=+0.5$ & Antiparallel & 100.43 & 6.036 & 0.3523 & Growth \\
$f / S=+5$ & Antiparallel & 35.49 & 5.901 & -0.2161 & Decay \\
\hline \hline
\end{tabular}




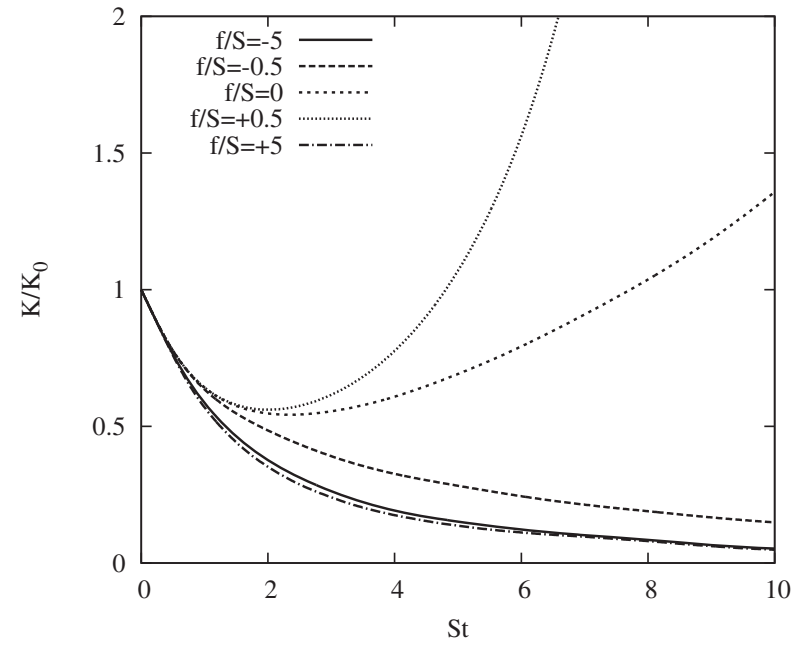

FIG. 2. Evolution of the turbulent kinetic energy $K$ in nondimensional time $S t$ for different rotation ratios $f / S$.

that shear has a more direct influence on the time scale of the overturning turbulent eddies as compared to rotation. Typical flow parameters are summarized in Table I for five selected cases taken at nondimensional time $S t=5$.

\section{RESULTS}

In this section, the turbulence evolution is first discussed. Then, the anisotropy properties of the turbulence are characterized using well-established measures in order to provide a reference point for the interpretation of newly defined quantities. Turbulence structure anisotropy tensors are discussed. Wavelet-based measures are used to obtain further information about the anisotropy properties of homogeneous turbulence with mean shear and system rotation. Finally, scaledependent statistics are used to quantify the intermittency and to obtain insight into geometrical features of the flow.

\section{A. Turbulence evolution}

Figure 2 shows the evolution of the turbulent kinetic energy $K$ for a series of simulations in which the rotation ratio $f / S$ is varied. Due to the isotropic initial conditions, the turbulent kinetic energy first decays in order to adjust to the flow anisotropy. The nonrotating case with $f / S=0$ shows eventual exponential growth of $K$. For moderate rotation ratios, the antiparallel case with $f / S=+0.5$ leads to a strong growth of the turbulent kinetic energy, while the parallel case with $f / S=-0.5$ results in a decay of $K$. For strong rotation ratios, however, both the antiparallel case with $f / S=+5$ and the parallel case with $f / S=-5$ lead to a strong decay of $K$ due to the importance of linear effects. These observations are in agreement with previous results (Refs. 2-4 and 7). A first rough classification of the different flows can thus be made by separating flows in which the energy eventually increases from flows in which the energy decays. One of the main goals of this paper is to see whether this can be related to other features of the flow.

A first step is to separately investigate the contributions

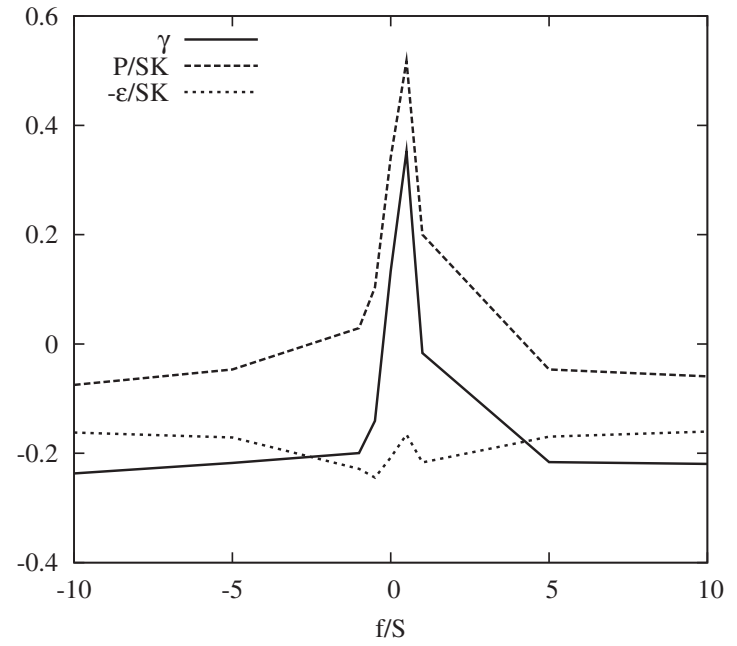

FIG. 3. Dependence of the growth rate $\gamma$, normalized production $P / S K$, and normalized dissipation $\epsilon / S K$ on the rotation ratio $f / S$ at nondimensional time $S t=5$.

of production and dissipation in the transport equation for the turbulent kinetic energy. This equation can be written in the following nondimensional form:

$$
\gamma=\frac{1}{S K} \frac{d K}{d t}=\frac{P}{S K}-\frac{\epsilon}{S K} .
$$

Here $\gamma$ is the growth rate of the turbulent kinetic energy, $P / S K$ is the normalized production rate, and $\epsilon / S K$ is the normalized dissipation rate. The dependence of the turbulence growth rate $\gamma$ on the rotation ratio $f / S$ is shown in Fig. 3 at nondimensional time $S t=5$ (see also Table I). Positive values of $\gamma$ correspond to growth of turbulent kinetic energy $K$ and negative values of $\gamma$ correspond to its decay. In accordance with the previous works, ${ }^{2-4,7}$ the antiparallel configuration with $0<f / S<1$ results in a destabilization of the flow, while other parameter ranges of the rotation ratio lead to a stabilization of the turbulence level. Both normalized production $P / S K$ and normalized dissipation $\epsilon / S K$ contribute to the growth rate $\gamma$. While the normalized dissipation rate remains relatively unaffected by a variation of the rotation ratio, the normalized production rate strongly increases in the antiparallel case with $0<f / S<1$, which leads to a fast growth of the turbulence. The mechanism that is responsible for the turbulent kinetic energy growth mainly acts at the large scales. We will come back to this issue in Secs. III D and III E, where we will analyze the flows using scale dependent statistics.

In order to investigate the effect of shear and rotation on the structure of turbulent flows, volume visualizations of the magnitude of fluctuating vorticity are considered (for details on volume visualization, see Ref. 23). Figures 4 and 5 show vortical structures for two cases with $f / S=+0.5$ and $f / S$ $=+5$, respectively, at nondimensional time $S t=5$. In the following, we will often take these two cases as representative for the two distinct energy evolution regimes. The vortical structures are inclined in the vertical direction relative to the downstream direction by an angle $\alpha$. This angle is larger for the strongly growing case with $f / S=+0.5$ compared to the 


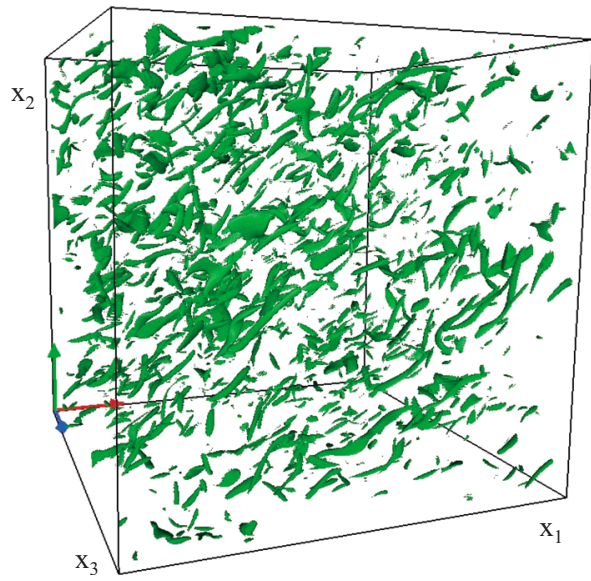

FIG. 4. (Color online) Volume visualization of isovorticity for $f / S=+0.5$ at nondimensional time $S t=5$. Orientation as in Fig. 1.

decaying case with $f / S=+5$. The inclination angle $\alpha$ of vortical structures directly influences the strength of turbulence growth or decay. In the decaying case with $f / S=+5$, the vortical structures are patchy and somewhat resemble structures found in stratified flows. The flow seems to be close to a two-component flow, such as in stably stratified turbulence, rather than to a two-dimensional flow. ${ }^{24}$ Using rapid distortion theory, Salhi ${ }^{9}$ pointed out similarities between rotation and stratification effects in homogeneous shear flow. Similarly, Kassinos et al. ${ }^{10}$ provided some insight into the interplay of rotation and stratification. In the case $f / S=+0.5$, the aligned structures are more elongated, indicating a partial two-dimensionalization of the flow. A more detailed discussion of the inclination angle $\alpha$ can be found in Jacobitz et al. ${ }^{8}$

\section{B. Conventional anisotropy measures}

Two conventional measures for the anisotropy properties of turbulent flow are computed from the direct numerical simulation data. The Reynolds stress anisotropy tensor $b_{i j}$ describes the large scale anisotropy properties

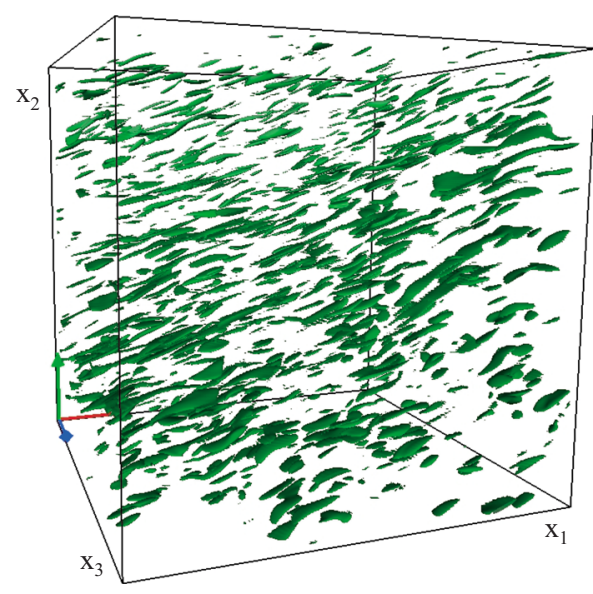

FIG. 5. (Color online) Volume visualization of isovorticity for $f / S=+5$ at nondimensional time $S t=5$. Orientation as in Fig. 1.

$$
b_{i j}=\frac{\overline{u_{i} u_{j}}}{2 K}-\frac{1}{3} \delta_{i j}
$$

The top left part of Fig. 6 shows the dependence of the diagonal components of the Reynolds shear stress anisotropy tensor $b_{i j}$ on the rotation ratio $f / S$ at nondimensional time $S t=5$. The diagonal components of $b_{i j}$ correspond to the distribution of energy among the velocity components. For most rotation ratios, an ordering $b_{11}>b_{33}>b_{22}$, i.e., streamwise $>$ spanwise $>$ vertical, is observed. Only in the antiparallel cases with $0<f / S<1$ this ordering is changed into $b_{22}$ $>b_{33}>b_{11}$, i.e., vertical $>$ spanwise $>$ streamwise. The offdiagonal components of $b_{i j}$ are shown at the bottom left part of Fig. 6. Due to the symmetry of the flow, the components $b_{13}$ and $b_{23}$ remain small. The magnitude of the component $b_{12}$ is largest for $f / S=+0.5$, corresponding to the strongest growth of the turbulent kinetic energy $K$. Note that the normalized turbulence production rate is related to the anisotropy features of the flow since $P / S K=-2 b_{12}$.

The dissipation rate anisotropy tensor $e_{i j}$ is defined in a similar manner to describe the small scale anisotropy properties of the flow

$$
e_{i j}=\frac{\nu \overline{\frac{\partial u_{i}}{\partial x_{k}} \frac{\partial u_{j}}{\partial x_{k}}}}{2 \epsilon}-\frac{1}{3} \delta_{i j} .
$$

The top right part of Fig. 6 shows the dependence of the diagonal components of the dissipation rate anisotropy tensor $e_{i j}$ on the rotation ratio $f / S$ at nondimensional time $S t=5$. Both the streamwise $e_{11}$ and spanwise $e_{33}$ components for most cases show a surplus, while the vertical component $e_{22}$ shows a deficit. Only in the strongly growing case with rotation ratio $f / S=0.5$, the ordering is altered. The off-diagonal components of $e_{i j}$ are shown at the bottom right part of Fig. 6. Again, only the $e_{12}$ component is nonzero. Overall, the components of the dissipation rate anisotropy tensor $e_{i j}$ closely follow the components of the Reynolds stress anisotropy tensor $b_{i j}$. The main drawback of the dissipation rate anisotropy tensor is the summation over all three gradients of velocity in each component. It is therefore not possible to capture additional directional information about this flow not already described in the Reynolds stress anisotropy tensor.

In order to gain more understanding about directional information contained in the velocity gradients, the contribution of each gradient to the overall dissipation rate is considered

$$
\epsilon_{i, j}=\frac{\nu \overline{\frac{\partial u_{i}}{\partial x_{j}} \frac{\partial u_{i}}{\partial x_{j}}}}{2 \epsilon} .
$$

In contrast to the dissipation rate anisotropy tensor, this alternative quantity does not require any summation over the spatial derivatives of the velocity components. The top row in Fig. 9 shows the dependence of $\epsilon_{i, j}$ on the rotation ratio $f / S$ at nondimensional time $S t=5$. The left figure shows the three gradients of the streamwise velocity component $\epsilon_{1, j}$. For most values of the rotation ratio, the vertical component 

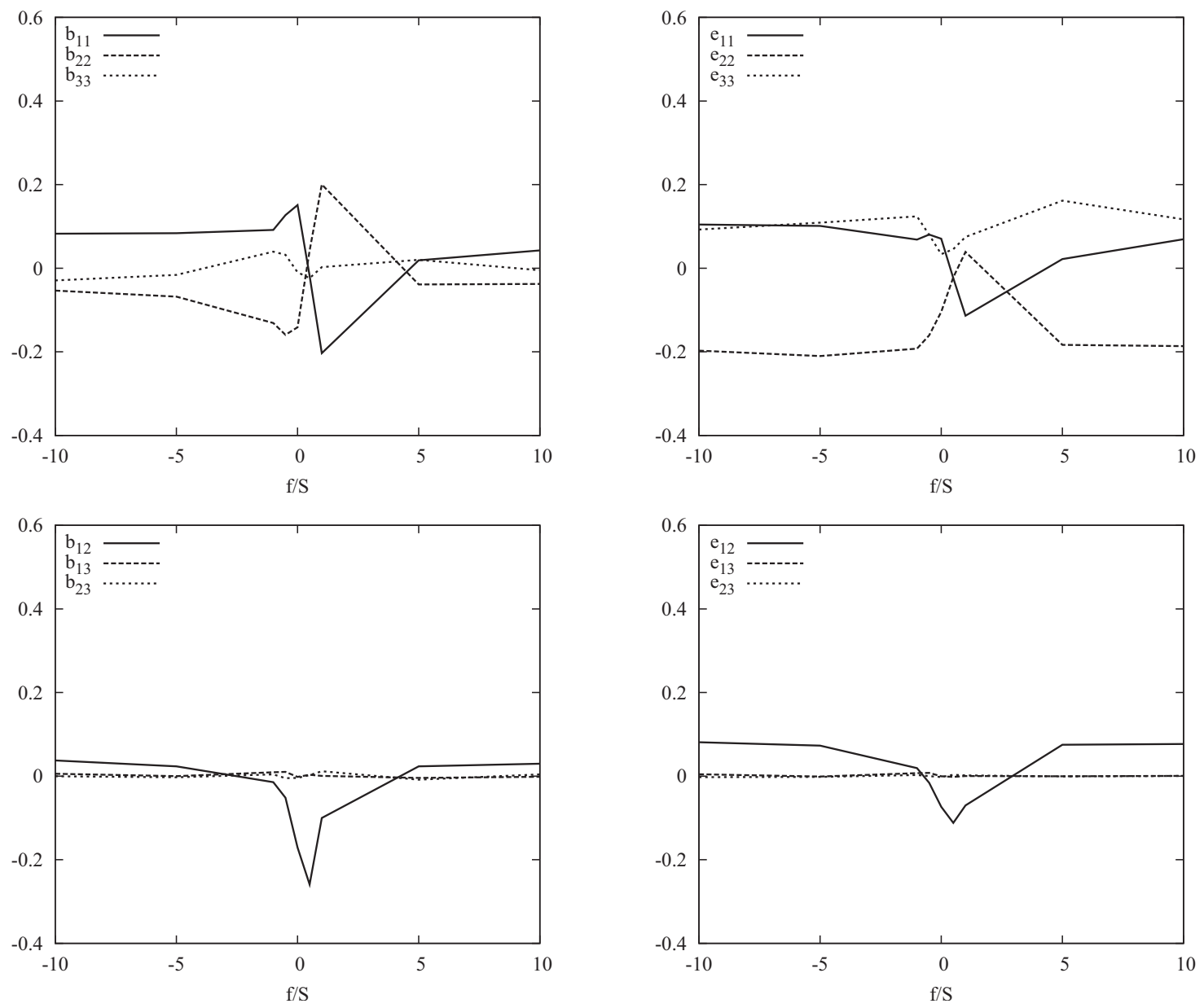

FIG. 6. Dependence of the diagonal components (top row) and off-diagonal components (bottom row) of the Reynolds stress anisotropy tensor $b_{i j}$ (left column) and the dissipation rate anisotropy tensor $e_{i j}$ (right column) on the rotation ratio $\mathrm{f} / \mathrm{S}$ at nondimensional time $S t=5$.

is the largest. It is, however, strongly reduced for the strongly growing cases with $0<f / S<1$, where the spanwise component becomes important. The center figure shows the gradients of the vertical velocity component $\epsilon_{2, j}$. Again, the spanwise gradient is strongly increased for the cases with strongly growing turbulent kinetic energy. The right figure shows the gradients of the spanwise velocity component $\epsilon_{3, j}$. For most cases, the vertical gradient shows the largest contribution, but it is reduced for the cases with strongly growing turbulent kinetic energy. In general, by magnitude large rotation ratios lead to large spanwise gradients of the velocity components. The cases with growing turbulent kinetic energy, however, are characterized by strong vertical gradients of the velocity components.

\section{Turbulence structure anisotropy tensors}

In order to gain a more complete description of the structure of turbulence, we consider decompositions of the Reynolds stress anisotropy tensor. For homogeneous turbulence, following Kassinos et al. ${ }^{20}$ the structure dimensionality tensor $D_{i j}$ can be determined from the velocity spectrum tensor $E_{i j}(\boldsymbol{k})=\overline{\hat{u}_{i} \hat{u}_{j}^{*}}$,

$$
D_{i j}=\int \frac{k_{i} k_{j}}{k^{2}} E_{n n}(\boldsymbol{k}) d^{3} \boldsymbol{k} .
$$

Here, a hat denotes the Fourier transform, a star the complex conjugate, and $\boldsymbol{k}=\left(k_{1}, k_{2}, k_{3}\right)$ is the wave vector. The structure dimensionality anisotropy tensor is then defined as follows:

$$
d_{i j}=\frac{D_{i j}}{D_{k k}}-\frac{1}{3} \delta_{i j}
$$

Another measure introduced by Kassinos et al. ${ }^{20}$ is the circulicity tensor

$$
F_{i j}=\int \frac{\overline{\hat{\omega}_{i} \hat{\omega}_{j}^{*}}}{k^{2}} d^{3} \boldsymbol{k}
$$

Here, $\boldsymbol{\omega}=\boldsymbol{\nabla} \times \boldsymbol{u}=\left(\omega_{1}, \omega_{2}, \omega_{3}\right)$ is the vorticity vector and $k$ is the magnitude of the wave vector. The circulicity anisotropy tensor is then defined as follows: 

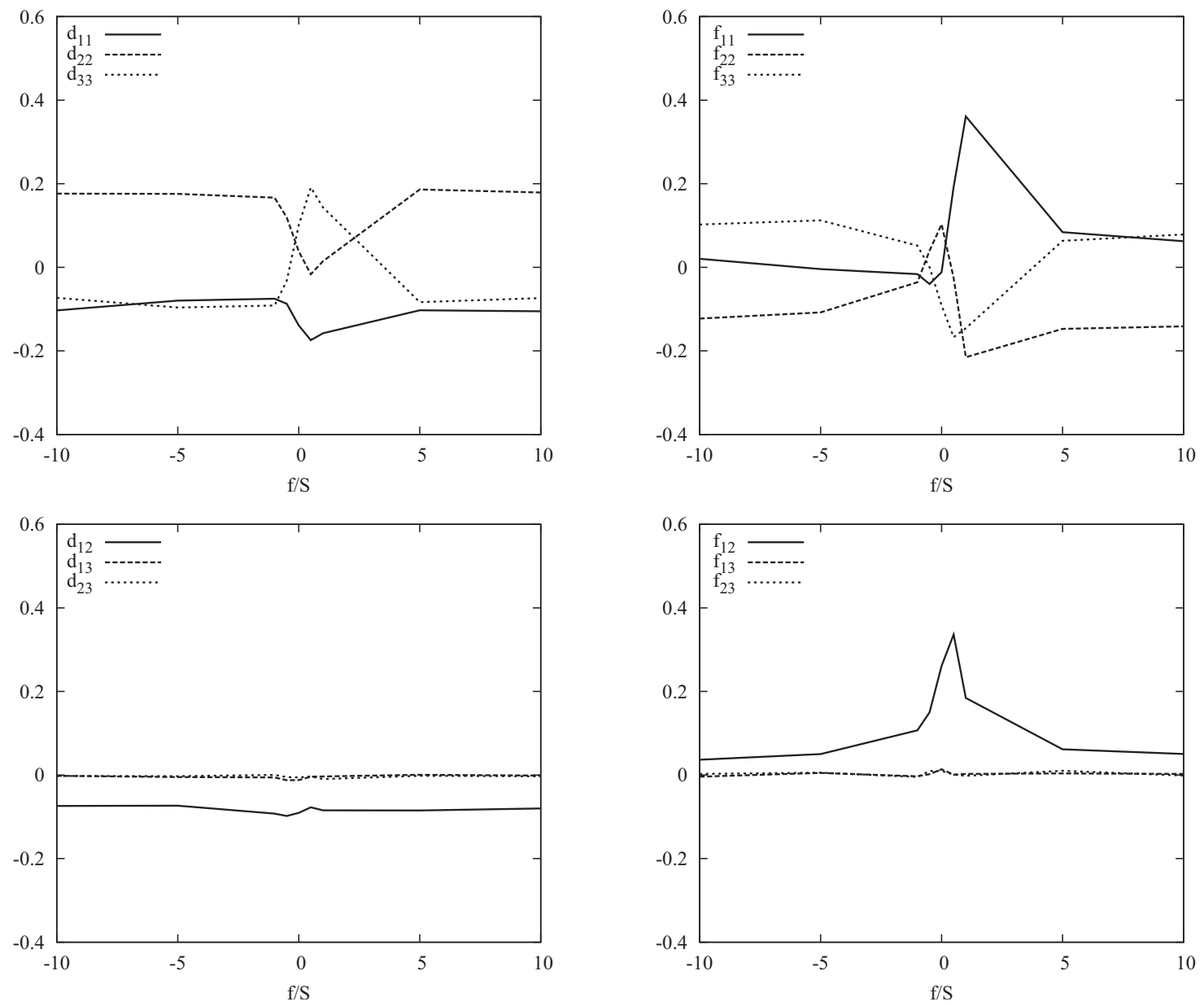

FIG. 7. Dependence of the diagonal components (top row) and off-diagonal components (bottom row) of the structure dimensionality anisotropy tensor $d_{i j}$ (left column) and the circulicity anisotropy tensor $f_{i j}$ (right column) on the rotation ratio $f / S$ at nondimensional time $S t=5$.

$$
f_{i j}=\frac{F_{i j}}{F_{k k}}-\frac{1}{3} \delta_{i j}
$$

The Reynolds stress anisotropy tensor, structure dimensionality anisotropy tensor, and circulicity anisotropy tensors are related,

$$
b_{i j}+d_{i j}+f_{i j}=0 .
$$

Note that the circulicity anisotropy tensor is therefore determined by the Reynolds stress anisotropy tensor and the dimensionality anisotropy tensor.

The left column of Fig. 7 shows the diagonal (top) and off-diagonal (bottom) components of the structure dimensionality anisotropy tensor. For the case of homogeneous shear flow with $f / S=0$, our results are consistent with those reported by Kassinos et al. ${ }^{20}$ In this case, we observe that $d_{22} \approx d_{33}>d_{11}$, which, according to Kassinos et al. ${ }^{20}$ suggests that "the dimensionality is close to being axisymmetric about the $x_{1}$-axis." For $f / S=+0.5$, an ordering $d_{33}>d_{22}$ $>d_{11}$ is obtained. Otherwise, we observe $d_{11} \approx d_{33}<d_{22}$. For the off-diagonal components, we observe that $d_{13}$ and $d_{23}$ vanish, while the component $d_{12}$ assumes a value of about -0.08 , independent of the rotation ratio $f / S$. This value again is in agreement with Kassinos et al. ${ }^{20}$
The right column of Fig. 7 shows the diagonal (top) and off-diagonal (bottom) components of the circulicity anisotropy tensor, which "describes the large-scale structure of the vorticity field. ${ }^{, 20}$ The nonzero values of $f_{12}$ confirm that the flow structures are inclined to the streamwise direction. For growing turbulent kinetic energy, $f_{11}$ is the dominant component. For negative $f / S$, we find $f_{33}>f_{11}>f_{22}$ and for $f / S$ $>5$, we observe $f_{11} \approx f_{33}>f_{22}$.

The corresponding wavenumber-dependent quantities can be defined by shell averaging in Fourier space and yield insight into the wavenumber distribution of different anisotropy tensors. Figure 8 shows the wavenumber-dependent components of the Reynolds stress anisotropy tensor $b_{i j}$ (left column), the structure dimensionality tensor $d_{i j}$ (center column), and the circulicity anisotropy tensor $f_{i j}$ (right column) for two values of the rotation ratios $f / S=+0.5$ (top row) and $f / S=+5$ (bottom row) at nondimensional time $\mathrm{St}=5$. Those two cases were chosen to illustrate both energy-growing and energy-decaying cases. The main conclusion inspecting these plots is that the flows show no return to isotropy at large wavenumber for all quantities and all flows. In addition, we find a pronounced anisotropy at small wavenumbers and a strong dependence of the values of the turbulence structure 

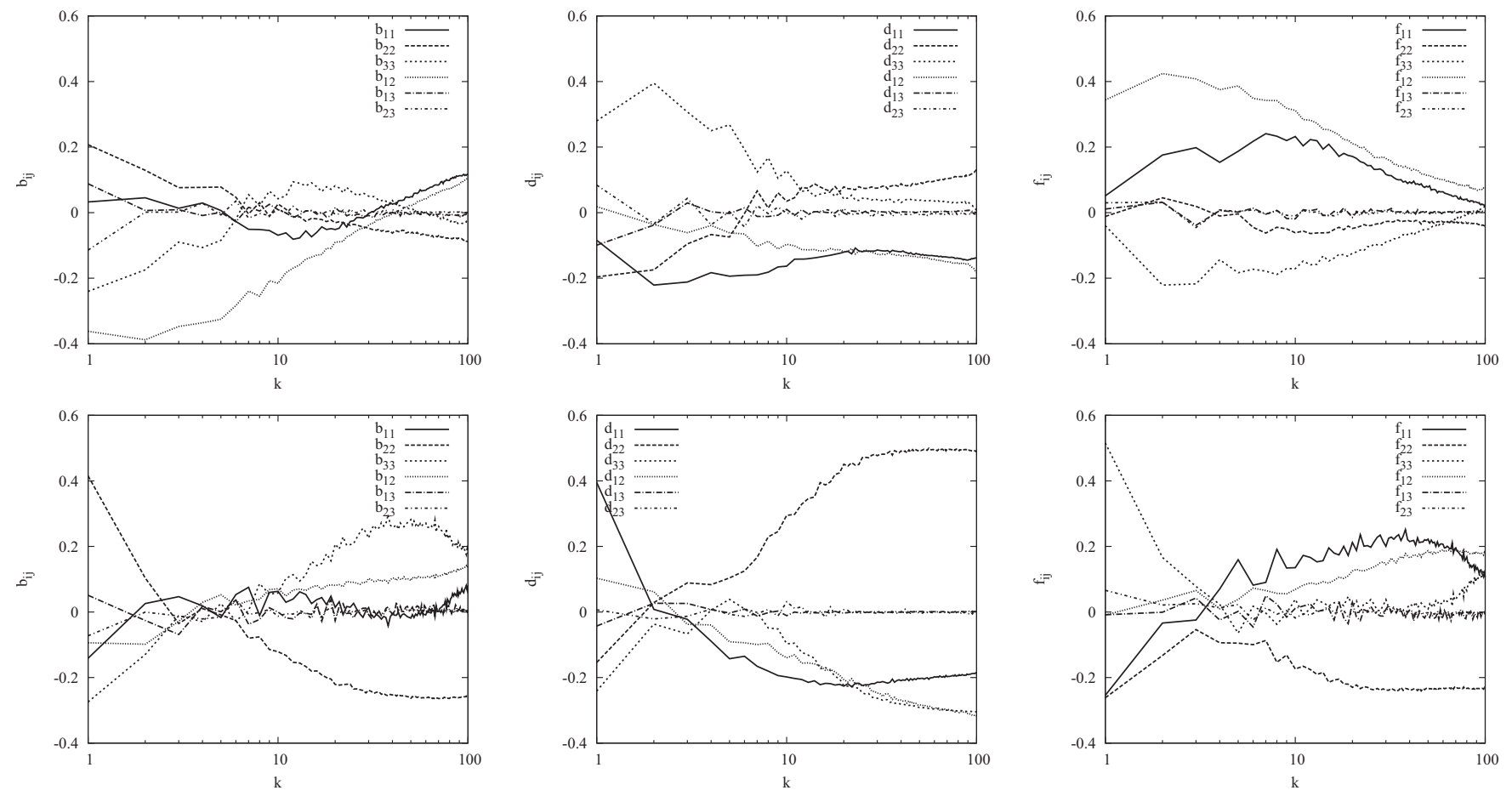

FIG. 8. Wavenumber dependence components of the Reynolds stress anisotropy tensor $b_{i j}$ (left column), structure dimensionality anisotropy tensor $d_{i j}$ (center column), and circulicity anisotropy tensor $f_{i j}$ (right column) for two cases with $f / S=+0.5$ (top row) and $f / S=+5$ (bottom row) at nondimensional time $S t=5$.

anisotropy tensors on the wavenumber. It remains to be verified if return to isotropy is observed at higher Reynolds numbers.

Based on an energy, polarization, and helicity decomposition of the three-dimensional energy spectrum tensor $E_{i j}$, Cambon et al. ${ }^{18}$ suggested a decomposition of the Reynolds stress anisotropy tensor $b_{i j}$ into directional anisotropy $b_{i j}^{(e)}$ and polarization anisotropy $b_{i j}^{(z)}$,

$$
b_{i j}=b_{i j}^{(e)}+b_{i j}^{(z)} .
$$

The structure dimensionality anisotropy tensor $d_{i j}$ and the circulicity anisotropy tensor $f_{i j}$ can be obtained as a linear combination of $b_{i j}^{(e)}$ and $b_{i j}^{(z)}$,

$$
d_{i j}=-2 b_{i j}^{(e)}, \quad f_{i j}=b_{i j}^{(e)}-b_{i j}^{(z)} .
$$

The authors ${ }^{18}$ showed that the directional anisotropy tensor is correctly determined by linear theory.

\section{Wavelet-based anisotropy measures}

Space-scale decomposition of the flow is obtained by applying the orthogonal wavelet transform to the velocity field. Therefore, the velocity field $\boldsymbol{u}=\left(u_{1}, u_{2}, u_{3}\right)$ at a given time instant is developed into an orthogonal wavelet basis using Coiflet 12 wavelets. ${ }^{25}$ Note that the same decomposition can be applied to the vorticity field $\boldsymbol{\omega}=\nabla \times \boldsymbol{u}$ $=\left(\omega_{1}, \omega_{2}, \omega_{3}\right)$. The projection of one component $u_{\alpha}(\mathbf{x})$ can be represented by

$$
u_{\alpha}(\mathbf{x})=\sum_{\lambda} \widetilde{u}_{\lambda}^{\alpha} \psi_{\lambda}(\mathbf{x})
$$

with the subscript $\lambda=(j, \mathbf{i}, d)$, where $j$ represents the scale index, $\mathbf{i}$ the position, and $d$ the direction. The orthogonal wavelet coefficients are given by $\widetilde{u}_{\lambda}^{\alpha}=\left\langle u_{\alpha}, \psi_{\lambda}\right\rangle$, where $\langle$,$\rangle de-$ notes the $L^{2}$-inner product. The wavelet coefficients measure the fluctuations of $u_{\alpha}$ at scale $2^{-j}$ and around position $\mathbf{i} / 2^{j}$ for each of the seven possible directions $d$. The contribution of the velocity component $u_{\alpha}$ at scale $2^{-j}$ and direction $d$ is obtained by fixing $j$ and $d$ and summing only over $\mathbf{i}$ in Eq. (14) and it is denoted by $u_{\alpha}^{j, d}$. Its contribution $u_{\alpha}^{j}$ at scale $2^{-j}$ is obtained by summation over $\mathbf{i}$ and $d$ in Eq. (14) while fixing $j$.

Parseval's identity allows one to obtain directional energy contributions as functions of scale $j .{ }^{14}$ For the directional scale-dependent energy distribution of a velocity component $u_{\alpha}$, we thus obtain

$$
E_{\alpha}^{j, d}=\frac{1}{2}\left\langle u_{\alpha}^{j, d}, u_{\alpha}^{j, d}\right\rangle
$$

Summing over all scales, we get the directional energy of the velocity component $u_{\alpha}$ in the direction $d$,

$$
E_{\alpha}^{d}=\sum_{j} E_{\alpha}^{j, d} .
$$

By construction, we obtain the total kinetic energy as follows: 

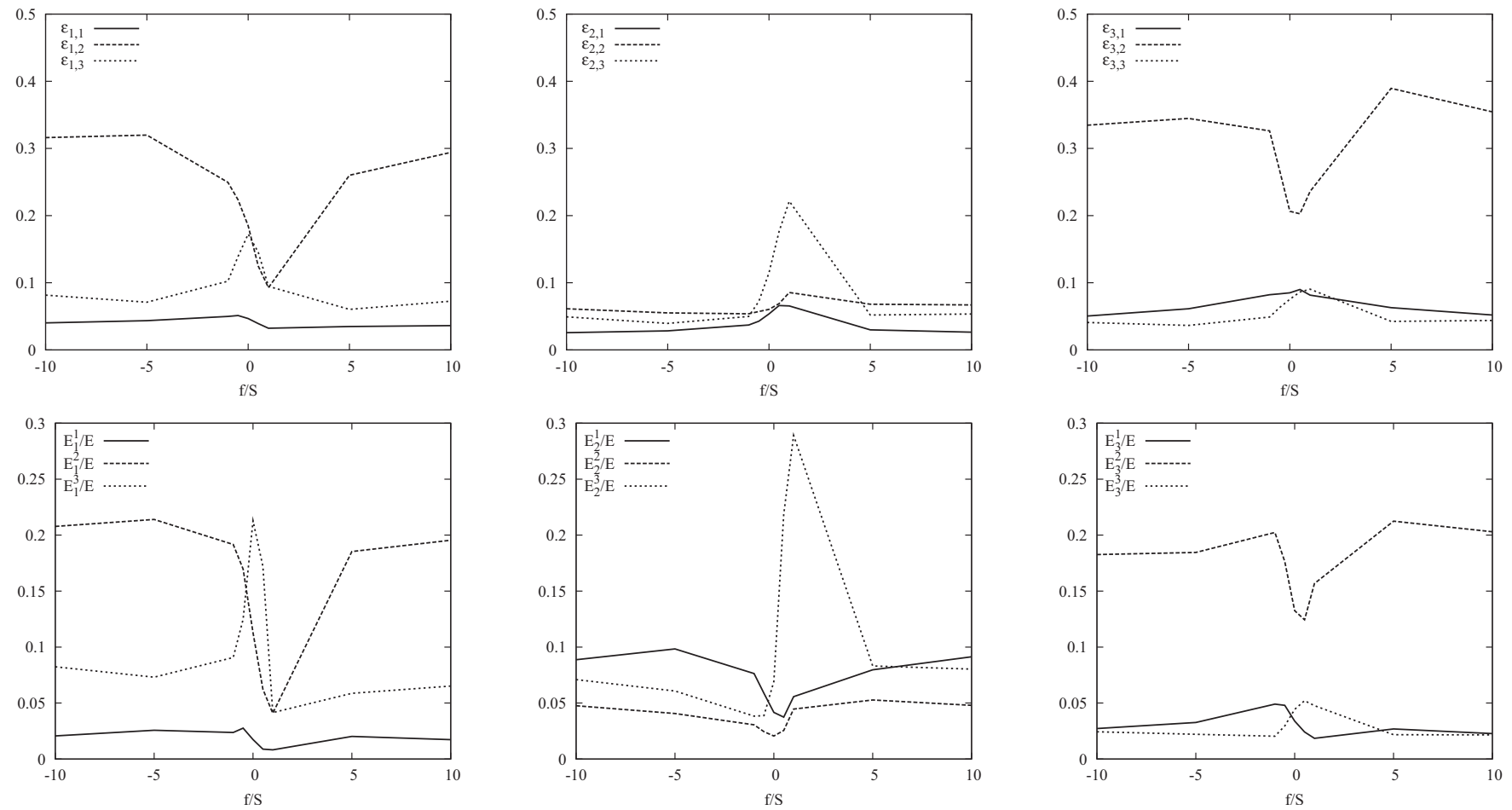

FIG. 9. Dependence of the dissipation rate component $\epsilon_{\alpha, \beta}$ for $\beta=1,2$ and 3 (top row) and directional energy component $E_{\alpha}^{d} / E$ for $d=1,2$, and 3 (bottom row) on the rotation ratio $f / S$ at nondimensional time $S t=5$. The $x_{1}$-component (left column), $x_{2}$-component (center column), and $x_{3}$-component (right column) are shown.

$$
E=\sum_{j, d, \alpha} E_{\alpha}^{j, d}=\sum_{d, \alpha} E_{\alpha}^{d}
$$

The bottom row in Fig. 9 shows the normalized directional energy components $E_{\alpha}^{d} / E$ for the $x, y$, and $z$ components. These wavelet-based measures exhibit a striking similarity with the normalized directional dissipation rate components $e_{i, j} / e$. This is due to the fact that wavelet coefficients of velocity are related to its gradients and discriminate the fluctuations of velocity components between the seven possible directions.

Figures 10 and 11 show the directional energy of the flow for two cases with $f / S=+0.5$ and $f / S=+5$, respectively, at nondimensional time $S t=5$. For the strongly growing case with $f / S=+0.5$, the vertical velocity $(v)$ in the spanwise di-

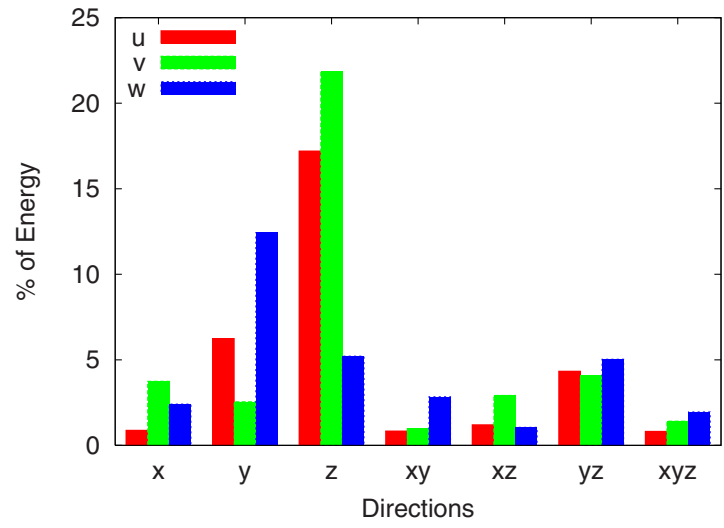

FIG. 10. (Color online) Wavelet-based directional energy for $f / S=+0.5$ at nondimensional time $S t=5$. rection $(z)$ contains most of the energy, followed by the downstream velocity $(u)$ in the spanwise direction $(z)$. For the strongly decaying case with $f / S=+5$, however, the spanwise $(w)$ and downstream $(u)$ velocities in the vertical direction (y) contain most of the energy, while vertical velocity $(v)$ is reduced. The figures also show that the mixed directions $(x y, x z, y z$, and $x y z)$ are less significant. A finer resolution of the anisotropy measures would require the use of the continuous wavelet transform, which necessitates substantially more computational resources for threedimensional turbulence. Details on this technique applied to two-dimensional cuts of three-dimensional turbulent flows can be found in Ruppert-Felsot et al. ${ }^{26}$

To study higher-order scale-dependent statistics, we de-

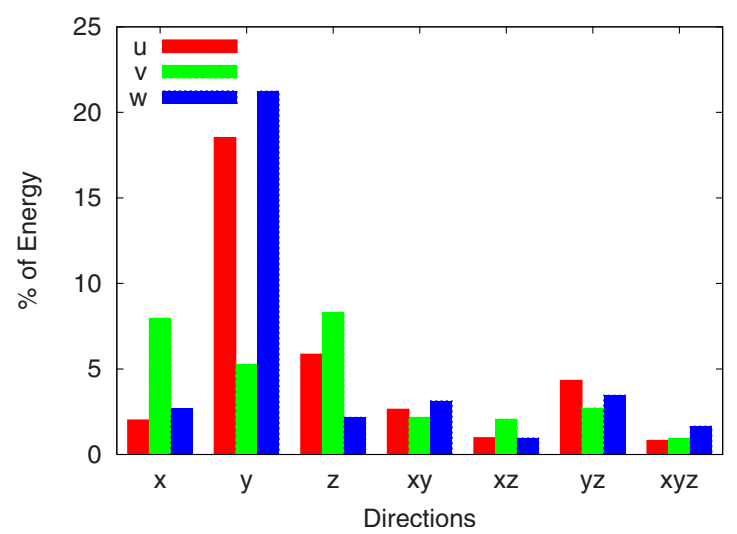

FIG. 11. (Color online) Wavelet-based directional energy for $f / S=+5$ at nondimensional time $S t=5$. 

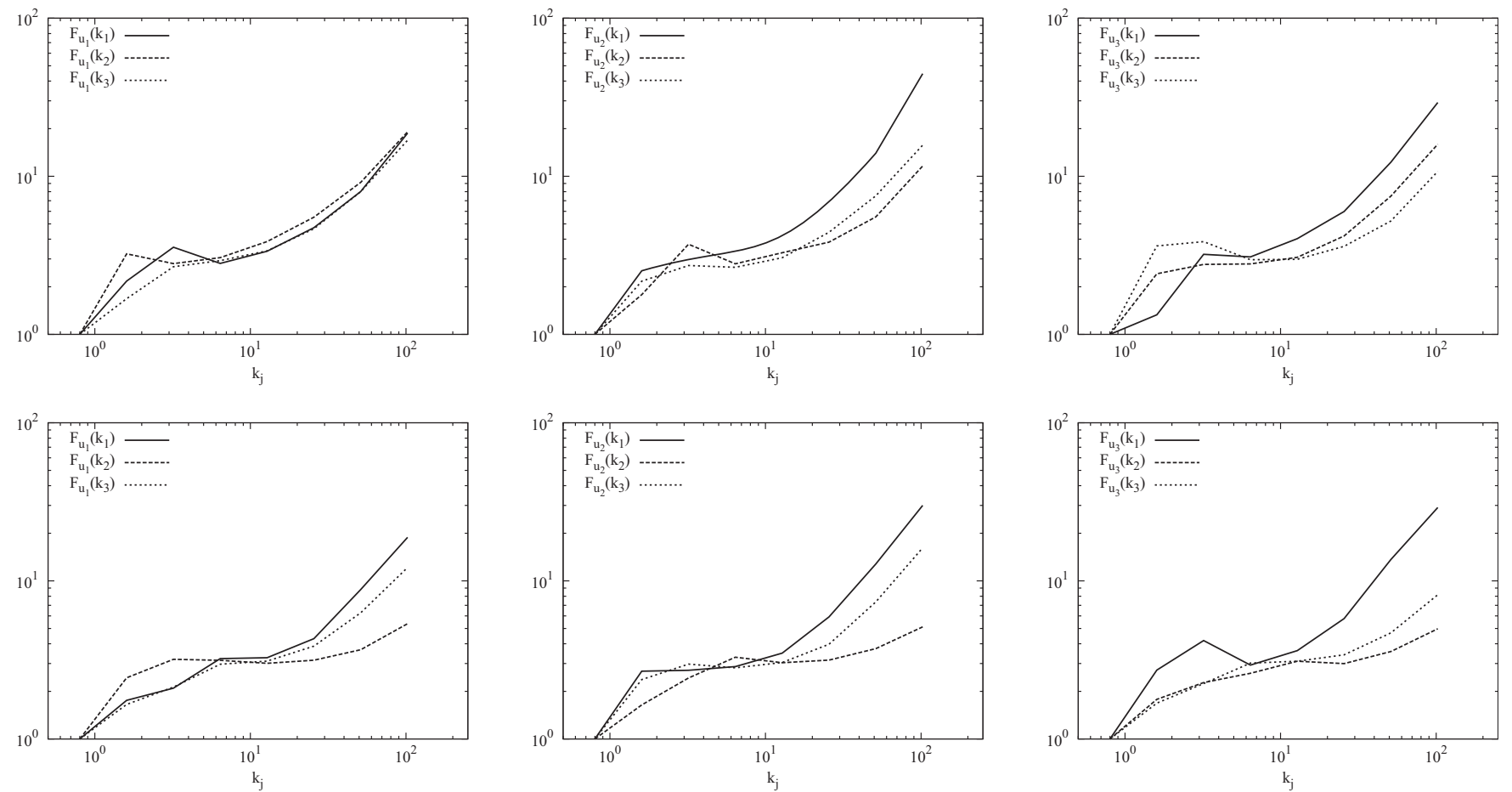

FIG. 12. Directional scale-dependent flatness for $f / S=+0.5$ (top row) and $f / S=+5$ (bottom row). The $u_{1}$-component (left column), $u_{2}$-component (center column) and $u_{3}$-component (right column) are shown.

fine the $p$-th order centered moments of each component $u_{\alpha}$ of the vector field $\mathbf{u}$ at scale $j$ from its wavelet coefficients (Ref. 27)

$$
M_{p, j}^{\alpha}=\frac{1}{7 \times 2^{3 j}} \sum_{i=0}^{2^{j}-1} \sum_{d=1}^{7}\left[\tilde{u}_{\lambda}^{\alpha}-\bar{M}_{j}^{\alpha}\right]^{p} .
$$

Here,

$$
\bar{M}_{j}^{\alpha}=\sum_{\mathbf{i}=0}^{2^{j}-1} \sum_{d=1}^{7} \tilde{u}_{\lambda}^{\alpha} /\left(7 \times 2^{3 j}\right),
$$

denotes the mean value of the moment at scale $j$. The scaledependent flatness of a velocity component $u_{\alpha}$ is defined as follows:

$$
F_{j}^{\alpha}=M_{4, j}^{\alpha}\left(M_{2, j}^{\alpha}\right)^{2}
$$

It is closely related to the standard deviation of the spectral distribution of energy, which illustrates that $F_{j}^{\ell}$ yields a measure of the relative spatial fluctuations of the spectral energy density. $^{14}$

The scale index $j$ is related to a wavenumber $k_{j}$ by the following relationship:

$$
k_{j}=k_{0} 2^{j}
$$

Here, $k_{0}$ is the centroid wavenumber of the mother wavelet, which is constant for each type of wavelet, e.g., $k_{0} \approx 0.77$ for the Coiflet 12 used here. Wavelets have a constant relative bandwidth, which means that with increasing $j$, the spectral support of the wavelet increases, and thus the spectral selectivity of the wavelet decreases. The scale-dependent distributions of energy or flatness can be related to wavenumber distributions, in particular, to energy spectra. ${ }^{25}$

The directional scale-dependent flatness of the three velocity components is shown in Fig. 12. Here, we focus only on two cases, one for $f / S=+0.5$ (top row), which is representative of a flow with strongly growing turbulent kinetic energy, and one for $f / S=+5$ (bottom row), which represents cases with decaying turbulent kinetic energy. A general observation is a strong increase of the flatness with wavenumber, which reflects the flow intermittency. The intermittency here is related to the dissipation range ${ }^{28}$ and the resolution of the simulations does not allow to observe a possible inertial range intermittency. In Ref. 14, it was shown that an anisotropy of the small spatial scales can cause an anisotropy of the directional flatness which is increased in certain directions due to the depletion of energy, affecting particular regions in Fourier space. Here, it is also observed that the small-scale intermittency is anisotropic. The growth of flatness of all velocity components is the strongest in the streamwise direction, except for the case $f / S=+0.5$ where the flatness of the $u_{1}$ velocity component behaves similarly in all directions due to the strong shear production. At moderate wavenumbers $2 \leq k \leq 10$, a flatness value around 3 is obtained in all cases, indicating a Gaussian-like behavior. Even though the flatness is slightly different for the two cases, it does not seem straightforward to relate the largescale production of kinetic energy directly to the directional intermittency of the flow. 


\section{E. Wavelet-based scale-dependent geometrical measures}

Additional geometrical information about the flow can be obtained from the velocity helicity $H_{u}$ and vorticity helicity $H_{\omega}$,

$$
H_{u}=\boldsymbol{u} \cdot(\nabla \times \mathbf{u}), \quad H_{\omega}=\boldsymbol{\omega} \cdot(\nabla \times \boldsymbol{\omega}) .
$$

After averaging over space, both quantities can be related by the helicity transport equation $d\left\langle H_{u}\right\rangle / d t=-2 \nu\left\langle H_{\omega}\right\rangle+2\langle F\rangle$. The term $F=\boldsymbol{f} \cdot \boldsymbol{\omega}$ accounts for any forcing terms $\boldsymbol{f}$ in the momentum equation, and it vanishes for linear effects, such as shear and rotation. A corresponding evolution equation for three-dimensional helicity spectrum was first given in Cambon and Jacquin. ${ }^{16}$ This equation also includes a transfer term, describing redistribution of helicity, determined by triple correlations. As in the case of the turbulent kinetic energy equation, the transfer term vanishes after averaging.

The present flow initially does not contain mean helicity and it will thus remain free from it. However, this does not concern the local helicity and regions with strong helicity can exist in a flow free from mean helicity. In the following, we will concentrate on this local helicity and its statistics.

The relative helicities of velocity and vorticity measure the cosine of the angle between the two vector quantities and are defined as follows:

$$
h_{u}=\frac{\boldsymbol{u} \cdot(\nabla \times \boldsymbol{u})}{\|\boldsymbol{u}\|\|\nabla \times \boldsymbol{u}\|}, \quad h_{\omega}=\frac{\boldsymbol{\omega} \cdot(\nabla \times \boldsymbol{\omega})}{\|\boldsymbol{\omega}\|\|\nabla \times \boldsymbol{\omega}\|} .
$$

The relative velocity helicity $h_{u}$ allows one to distinguish between helical structures (swirling motion) and nonhelical structures. For helical structures, $h_{u}$ has values of \pm 1 , which correspond to alignment or antialignment of vorticity and velocity, respectively. For nonhelical structures, twodimensionalization of the flow occurs, vorticity is perpendicular to velocity, and the velocity helicity $h_{u}$ assumes a value $h_{u}=0$. Using the vector identity $\boldsymbol{\nabla} \times \boldsymbol{\omega}=-\Delta \boldsymbol{u}$, the relative vorticity helicity $h_{\omega}$ measures the cosine of the angle between vorticity and the negative Laplacian of velocity, which is related to dissipation. The velocity helicity $h_{u}$ and vorticity helicity $h_{\omega}$ can also be interpreted as the correlation coefficients between $\boldsymbol{u}$ with $\boldsymbol{\omega}$ and $\boldsymbol{\omega}$ with $\boldsymbol{\nabla} \times \boldsymbol{\omega}$, respectively.

Scale-dependent helicities $h_{u^{j}}$ and $h_{\omega^{j}}$ can be defined by replacing $\boldsymbol{u}$ and $\boldsymbol{\omega}$ in Eq. (23) by $\boldsymbol{u}^{j}$ and $\boldsymbol{\omega}^{j}$, respectively, as recently introduced in Ref. 21. Thus, geometrical statistics can be obtained at different scales of the flow.

Figures 13 and 14 show the probability distribution functions (PDFs) of the relative helicities of velocity and vorticity, respectively. The PDFs of $h_{u}$ show a maximum for $h_{u}$ $=0$ for cases with growing turbulent kinetic energy $(f / S=0$ and $f / S=+0.5$ ), indicating a higher probability for twodimensional motion. For decaying cases, a maximum for $h_{u} \pm 1$ is observed, corresponding to a higher probability for helical motion. The PDFs of $h_{\omega}$ show a maximum for $h_{\omega} \pm 1$ for all cases and the alignment or antialignment of vorticity with the negative Laplacian of velocity is particularly pronounced in the case of strong rotation $(f / S= \pm 5)$.

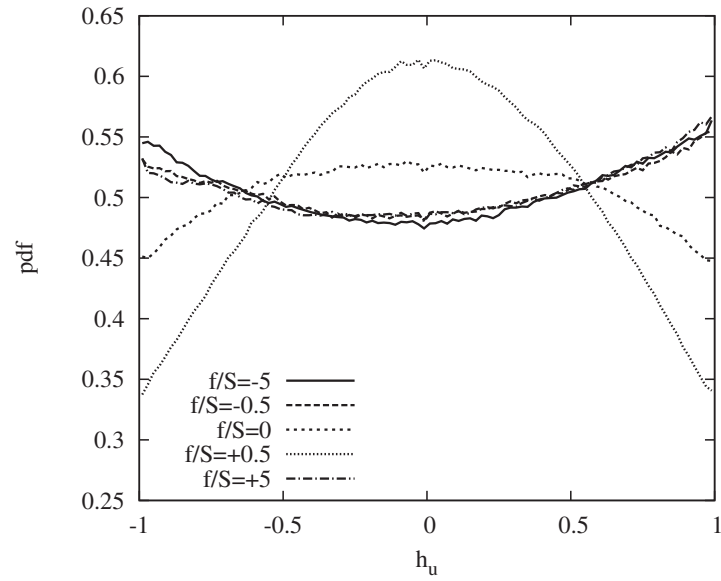

FIG. 13. Velocity helicity distribution $h_{u}$ at nondimensional time $S t=5$.

Figure 15 shows the scale-dependent velocity helicity PDF (left column) and vorticity helicity PDF (right column) for cases with $f / S=+0.5$ (top row) and $f / S=+5$ (bottom row) at nondimensional time $S t=5$. The scale-dependent velocity helicity PDFs confirm the significant difference between the two cases $f / S=+0.5$ and +5 , representing turbulence growth and decay, respectively, that we have mentioned above. For the former case, the PDFs show a pronounced twodimensional behavior at large scales $(j=3,4,5)$, while for the latter no peak around $h_{u^{j}}=0$ can be observed. The probability to have swirling motion at small scales is also more pronounced for $f / S=+5$. Similar conclusions hold for the scale-dependent distributions of vorticity helicity. These results clearly show that the mechanism which distinguishes the cases $f / S=+0.5$ and +5 is related to the large scales, as we have conjectured in Sec. III A. Apparently, in the $f / S$ $=+0.5$ case, the large scale flow shares some features with two-dimensional turbulence and the energy cascade is thus less efficient in transporting energy toward the small scales compared to three-dimensional turbulence.

Sanada $^{29}$ conjectured that $\left\langle H_{u}\right\rangle$ and $\left\langle H_{\omega}\right\rangle$ have a tendency to have the same sign. This tendency also holds for the corresponding pointwise quantities $H_{u}$ and $H_{\omega}$ (Ref. 30). If this conjecture holds, $H_{\omega}$ acts as a "kind of viscous dissipation"

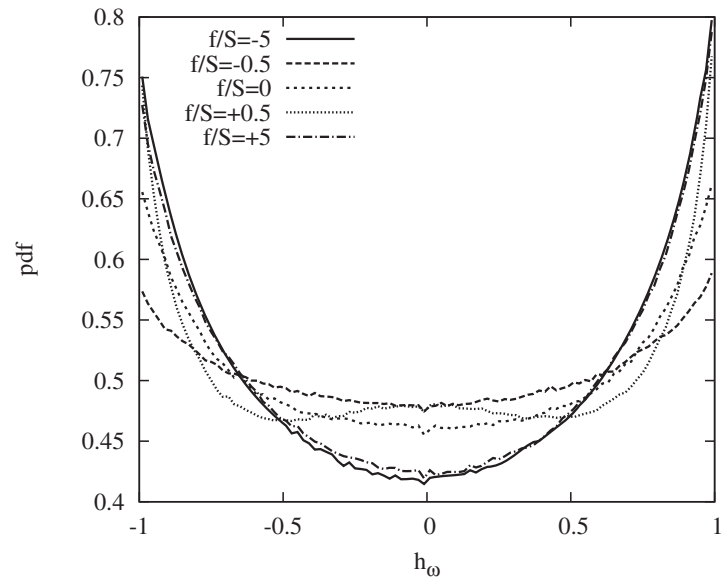

FIG. 14. Vorticity helicity distribution $h_{\omega}$ at nondimensional time $S t=5$. 

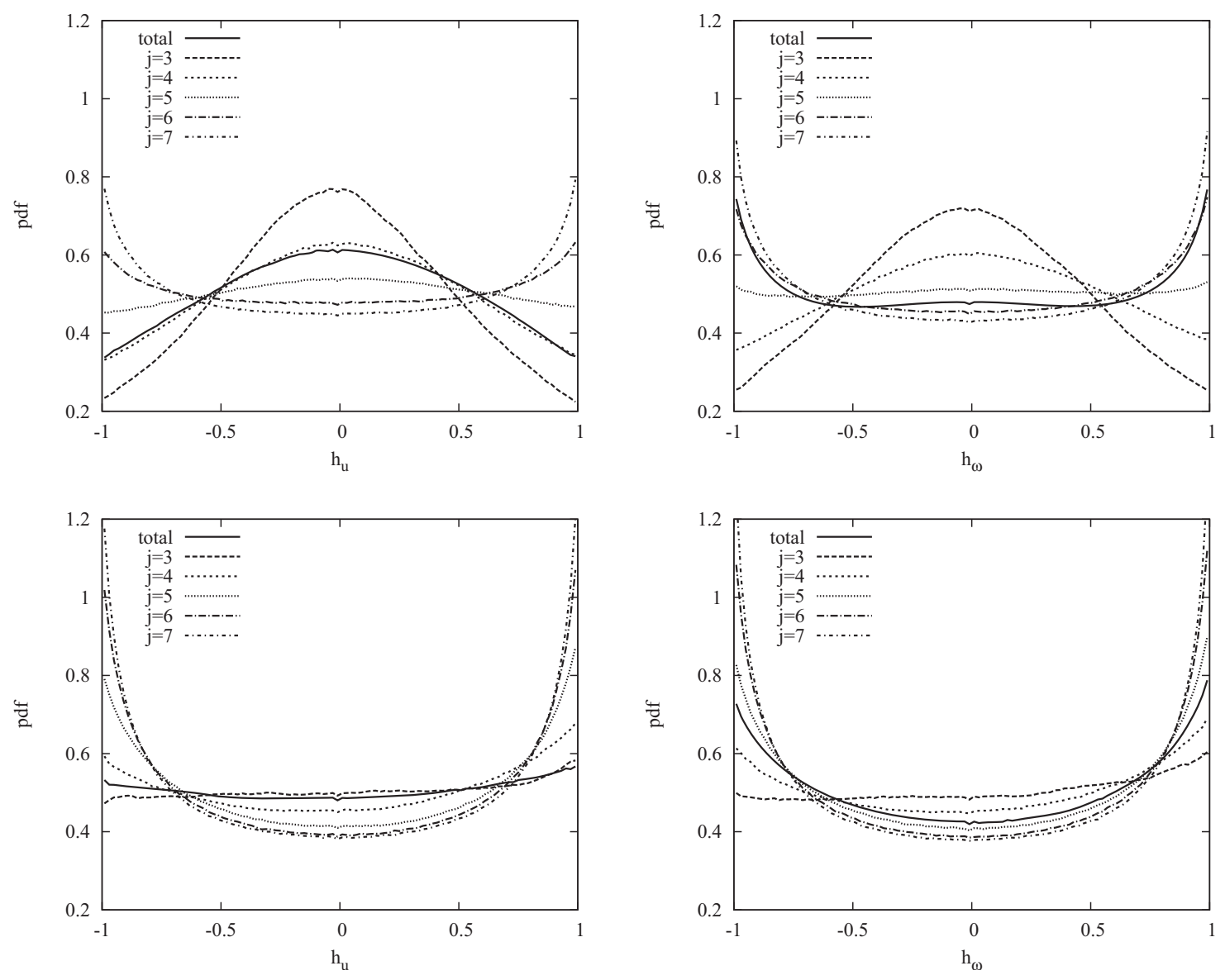

FIG. 15. Scale-dependent velocity helicity distribution $h_{u^{j}}$ (left column) and vorticity helicity distribution $h_{\omega^{j}}$ (right column) for $f / S=+0.5$ (top row) and for $f / S=+5$ (bottom row) at nondimensional time $S t=5$.

for $H_{u}$, independent of its sign. To verify Sanada's conjecture that both velocity helicity and vorticity helicity have the same sign, we show in Fig. 16 the joint probability distribution function $p\left(h_{u}, h_{\omega}\right)$. For all flows, the joint PDFs of $h_{u}$ and $h_{\omega}$ are not statistically independent. We find a higher probability in the two quadrants in which $h_{u}$ and $h_{\omega}$ have the same sign as in those with opposite signs. The highest probability is observed in lower left and upper right corners, corresponding to a high probability to find alignment or antialignment of $\boldsymbol{u}$ with $\boldsymbol{\omega}$ and $\boldsymbol{\omega}$ with $\boldsymbol{\nabla} \times \boldsymbol{\omega}$. This result supports the conjecture reported by Sanada ${ }^{29}$ and Galanti and Tsinober. ${ }^{30}$ For comparison, we also added the joint PDF for independently uniformly distributed velocity helicity and vorticity helicity fields, which results in a uniform joint PDF with mean value $1 / 4$.

\section{CONCLUSIONS}

Nine direct numerical simulations of homogeneous turbulence with shear and rotation have been performed and analyzed for this study. The turbulent kinetic energy was found to grow strongly in the antiparallel configuration with $0<f / S<1$ and to decay otherwise. The growth is due to an increased normalized turbulence production $P / S K=-2 b_{12}$ that is directly related to the only nonzero off-diagonal component of the Reynolds stress anisotropy tensor. It was also observed that the growth rate of the turbulence is related to the inclination angle of vortical structures relative to the downstream direction. ${ }^{8}$

The anisotropy of the flows is investigated with conventional measures, such as the Reynolds stress anisotropy tensor, the dissipation rate anisotropy tensor, and the normalized components of the dissipation rate. In addition, turbulence structure anisotropy tensors are considered and results for sheared turbulence are in agreement with Kassinos et al. ${ }^{20}$ The wavenumber-dependent tensors showed no return to isotropy at large wavenumbers. The directional energy of the flow, obtained from orthogonal wavelet decomposition of the velocity field, allows one to give an alternative measure of the anisotropy of the flow. Wavelets, such as structure functions, are sensitive to velocity differences in the different directions and allow to characterize streamwise, vertical, and spanwise anisotropy. Furthermore, orthogonal wavelets have the advantage that, due to their orthogonality, the energies contained in the different directions sum up to the total energy, unlike structure functions or one-dimensional spectra. It has been shown in this paper that these directional energies capture the properties of velocity gradients in rotating shear turbulence. For the strongly growing case with $f / S=+0.5$, the spanwise differences of vertical velocity contain most of the energy, followed by the spanwise differences of downstream velocity. For the strongly decaying case with $f / S$ 

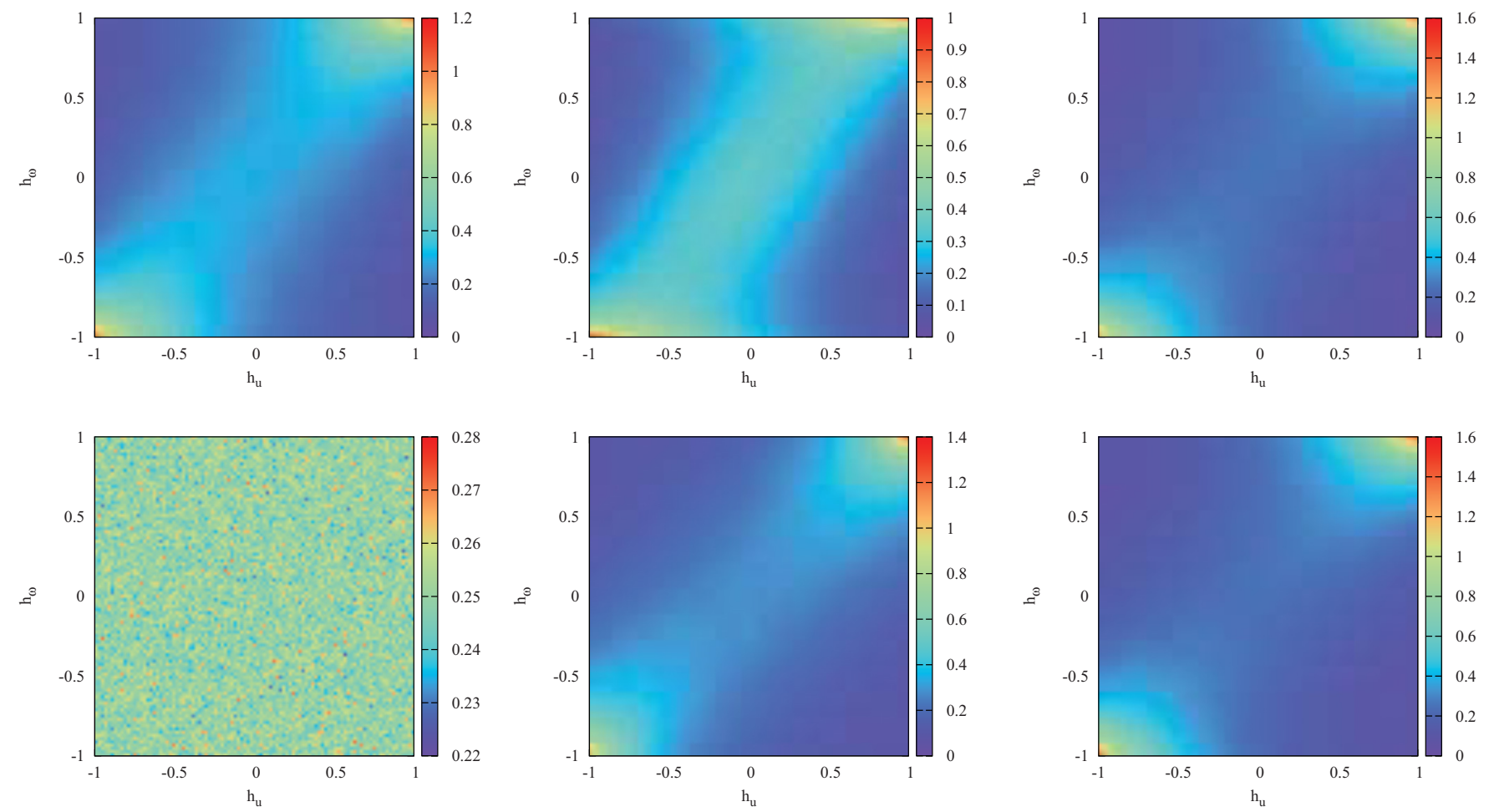

FIG. 16. (Color online) Joint PDFs of velocity helicity $h_{u}$ with vorticity helicity $h_{\omega}$ for cases with $f / S=0$ (top left), $f / S=+0.5$ (top center), $f / S=-0.5$ (bottom center), $f / S=+5$ (top right), $f / S=-5$ (bottom right), and uniformly distributed random fields (bottom left).

$=+5$, however, the vertical differences of spanwise and downstream velocities contain most of the energy, while vertical velocity is strongly reduced. It has thus been confirmed that wavelet-based directional energy measures agree with conventional measures.

The intermittency of the different flows studied here has been quantified using scale-dependent flatness in the different flow directions. Small-scale intermittency has been observed in most cases with an increased level of intermittency in the streamwise direction. At intermediate scales, a Gaussian-like behavior has been found. These observations confirm that the scale-dependent flatness is well suited to quantify the flow intermittency in different directions.

The geometrical statistics of the flows have been analyzed by considering PDFs of velocity helicity and of vorticity helicity. For the cases with growing turbulent kinetic energy, the local relative helicity indicates a tendency toward two-dimensional motion. However, for the decaying cases, helical motion (reflected by an alignment or antialignment of velocity and vorticity) is mainly observed. A scale-dependent study of helicity shows that for all cases small scales exhibit helical motion, while two-dimensionalization is observed at larger scales. It has thus been shown that the helicity of the flow strongly depends on the scale.

Joint PDFs of velocity helicity and vorticity helicity indicate a strong correlation of the signs of these quantities. This observation supports the conjectures reported by Sanada ${ }^{29}$ and Galanti and Tsinober. ${ }^{30}$ The results suggest that vorticity helicity tends to diminish velocity helicity for rotating and sheared turbulence.

\section{ACKNOWLEDGMENTS}

F.G.J. acknowledges support from the Ecole Centrale de Marseille, the Université de Provence, and an International Opportunity Grant from the University of San Diego. W.B., M.F., and K.S. acknowledge financial support from the ANR (Project No. M2TFP) and K.S. thanks the University of San Diego for its hospitality. M.F. is grateful to the Wissenschaftskolleg zu Berlin for its support. We thank B. Kadoch for the preparation of the volume visualizations.

\footnotetext{
${ }^{1}$ M. S. Miesch, "Large-scale dynamics of the convection zone and tachocline," Living Rev. Solar Phys. 2, 1 (2005).

${ }^{2} \mathrm{P}$. Bradshaw, "The analogy between streamline curvature and buoyancy in turbulent shear flow," J. Fluid Mech. 36, 177 (1969).

${ }^{3}$ D. J. Tritton, "Stabilization and destabilization of turbulent shear-flow in a rotating fluid," J. Fluid Mech. 241, 503 (1992).

${ }^{4}$ C. Cambon, J.-P. Benoit, L. Shao, and L. Jacquin, "Stability analysis and large-eddy simulation of rotating turbulence with organized eddies," J. Fluid Mech. 278, 175 (1994).

${ }^{5}$ S. Leblanc and C. Cambon, "On the three-dimensional instabilities of plane flows subjected to Coriolis force," Phys. Fluids 9, 1307 (1997).

${ }^{6}$ A. Salhi and C. Cambon, "An analysis of rotating shear flow using linear theory and DNS and LES results," J. Fluid Mech. 347, 171 (1997).

${ }^{7} \mathrm{G}$. Brethouwer, "The effect of rotation on rapidly sheared homogeneous turbulence and passive scalar transport. Linear theory and direct numerical simulations," J. Fluid Mech. 542, 305 (2005).

${ }^{8}$ F. G. Jacobitz, L. Liechtenstein, K. Schneider, and M. Farge, "On the structure and dynamics of sheared and rotating turbulence: Direct numerical simulation and wavelet-based coherent vortex extraction," Phys. Fluids 20, 045103 (2008).

${ }^{9}$ A. Salhi, "Similarities between rotation and stratification effects on homogeneous shear flow," Theor. Comput. Fluid Dyn. 15, 339 (2002).
} 
${ }^{10}$ S. C. Kassinos, E. Akylas, and C. A. Langer, "Rapidly sheared homogeneous stratified turbulence in a rotating frame," Phys. Fluids 19, 021701 (2007).

${ }^{11}$ S. C. Kassinos, B. Knaepen, and D. Carati, "The transport of a passive scalar in magnetohydrodynamic turbulence subjected to mean shear and frame rotation," Phys. Fluids 19, 015105 (2007).

${ }^{12}$ P. Sagaut and C. Cambon, Homogeneous Turbulence Dynamics (Cambridge University Press, Cambridge, 2008).

${ }^{13}$ C. G. Speziale and T. B. Gatski, "Analysis and modelling of anisotropies in the dissipation rate of turbulence," J. Fluid Mech. 344, 155 (1997).

${ }^{14}$ W. J. T. Bos, L. Liechtenstein, and K. Schneider, "Small scale intermittency in anisotropic turbulence," Phys. Rev. E 76, 046310 (2007).

${ }^{15} \mathrm{~K}$. Schneider and O. Vasilyev, "Wavelet methods in computational fluid dynamics," Annu. Rev. Fluid Mech. 42, 473 (2010).

${ }^{16}$ C. Cambon and L. Jacquin, "Spectral approach to non-isotropic turbulence subjected to rotation," J. Fluid Mech. 202, 295 (1989).

${ }^{17}$ C. Cambon, L. Jacquin, and J. L. Lubrano, "Toward a new Reynolds stress model for rotating turbulent flows," Phys. Fluids A 4, 812 (1992).

${ }^{18}$ C. Cambon, N. N. Mansour, and F. S. Godeferd, "Energy transfer in rotating turbulence," J. Fluid Mech. 337, 303 (1997).

${ }^{19}$ S. C. Kassinos and W. C. Reynolds, "A structure-based model for the rapid distortion of homogeneous turbulence," Stanford Report No. TF-61, 1994.

${ }^{20}$ S. C. Kassinos, W. C. Reynolds, and M. M. Rogers, "One-point turbulence structure tensors," J. Fluid Mech. 428, 213 (2001).

${ }^{21}$ K. Yoshimatsu, N. Okamoto, K. Schneider, Y. Kaneda, and M. Farge,
"Intermittency and scale-dependent statistics in fully developed turbulence," Phys. Rev. E 79, 026303 (2009).

${ }^{22}$ R. S. Rogallo, "Numerical experiments in homogeneous turbulence," NASA Report No. TM 81315, 1981.

${ }^{23}$ J. Clyne, P. Mininni, A. Norton, and M. Rast, "Interactive desktop analysis of high resolution simulations: Application to turbulent plume dynamics and current sheet formation," New J. Phys. 9, 301 (2007).

${ }^{24} \mathrm{C}$. Cambon, "Turbulence and vortex structures in rotating and stratified flows," Eur. J. Mech. B/Fluids 20, 489 (2001).

${ }^{25}$ M. Farge, "Wavelet transforms and their applications to turbulence," Annu. Rev. Fluid Mech. 24, 395 (1992).

${ }^{26}$ J. Ruppert-Felsot, M. Farge, and P. Petitjeans, "Wavelet tools to study intermittency: Application to vortex bursting," J. Fluid Mech. 636, 427 (2009).

${ }^{27}$ K. Schneider, M. Farge, and N. Kevlahan, "Spatial intermittency in twodimensional turbulence: A wavelet approach," in Woods Hole Mathematics: Perspectives in Mathematics and Physics, edited by N. Tongring and R. C. Penner (World Scientific, Singapore, 2004), Vol. 34, p. 302.

${ }^{28}$ R. H. Kraichnan, "Intermittency in the very small scales of turbulence," Phys. Fluids 10, 2080 (1967).

${ }^{29} \mathrm{~T}$. Sanada, "Helicity production in the transition to chaotic flow simulated by Navier-Stokes equation," Phys. Rev. Lett. 70, 3035 (1993).

${ }^{30} \mathrm{~B}$. Galanti and A. Tsinober, "Physical space properties of helicity in quasihomogeneous forced turbulence," Phys. Lett. A 352, 141 (2006). 